\title{
LCphysX: Teaching Undergraduate Physics Majors Multi- Media Science Communication Skills for Public Outreach
}

\author{
Shannon O’Leary* and Parvaneh Abbaspour† \\ *Department of Physics, Lewis \& Clark College, 0615 SW Palatine Hill Rd, Portland, OR 97219 \\ $†$ 'Aubrey R. Watzek Library, Lewis \& Clark College, 0615 SW Palatine Hill Rd, Portland, OR 97219
}

\begin{abstract}
L C$ phys $X$ is a web-based platform designed with the primary purpose of teaching undergraduate physics majors in upper-level laboratory courses multi-media science communication skills for engaging in public outreach. Compelling arguments have recently been made for the need to train future scientists to engage with a popular audience using digital media. Developed in partnership with Lewis \& Clark College's (LC) Library Digital Initiatives team, LCphys $X$ features short student-produced videos that present projects and experiments from upper-level lab courses to a popular audience. LC's unique Advanced Laboratory course provides students with the opportunity to design, build, and test their own physics projects, and for many students, their project is the main cumulative achievement of their physics education. Indexed, searchable videos on the LCphys $X$ website permanently archive LC physics projects and serve as pedagogical reference points and inspiration for physics faculty and students at LC and beyond. In addition, as the Advanced Laboratory course is a requirement for the LC physics major, all physics graduates will leave LC with an active "link" for their resumé that showcases their technical achievements, as well as their ability to communicate scientific ideas. Assessment of $L C p h y s X$ 's effectiveness in engaging the public will include feedback solicited from high school students across the United States.
\end{abstract}

Keywords: science communication, public outreach, electronic media, laboratory course design

PACS: $01.20 .+\mathrm{x}, 01.30 . \mathrm{Xx}, 01.50 . \mathrm{Qb}$

\section{INTRODUCTION}

For well-over a decade the ability to effectively communicate scientific information has been internationally recognized as a core competency for undergraduate science majors. ${ }^{1}$ Physics educators typically teach written communication through laboratory notebooks and journal-style laboratory reports, and approach oral communication in standard colloquium-style presentations that are geared toward audiences of undergraduate peers.

Emphasis on the importance of formally training students to communicate scientific information to nonscientific audiences has recently received increasing attention. ${ }^{2,3}$ In an era when public funding for science enters annual budget debates, and society must confront challenges on a scale that requires the enactment of national and international science-based policy, there is a strong need to teach the next generation of physicists why they should and how they can be strong advocates for science. Yet, neither the importance of outreach nor best practices in how to communicate with a public audience is currently a standard component of typical undergraduate physics curriculum.

Online video is increasingly becoming a popular medium for presentation of both formal and informal scientific information. For example, a growing number of academic science journals, like the openaccess New Journal of Physics, offer the option for authors to create video abstracts, and sites like the Journal for Visualized Experiments (JoVE) publishes entire peer-reviewed scientific videos alongside traditional papers. The German Research Foundation created an Internet video portal as a means of reaching the public interested in scientific research, and for inspiring German youth towards careers in science. ${ }^{4}$

For public outreach, online videos have several advantages. As the Internet has become a primary venue for information exchange, it is unmistakably the main platform on which scientific research is both sought by and channeled to the public. ${ }^{5}$ In an era of big data and information overload, if you want to reach people, it is necessary to engage them in forums they already frequent. ${ }^{3}$ The video format supports storytelling, which in the case of science communication means that scientific findings can be 
LCPhySX $\quad$ Projects what is this? Q

\section{Projects}

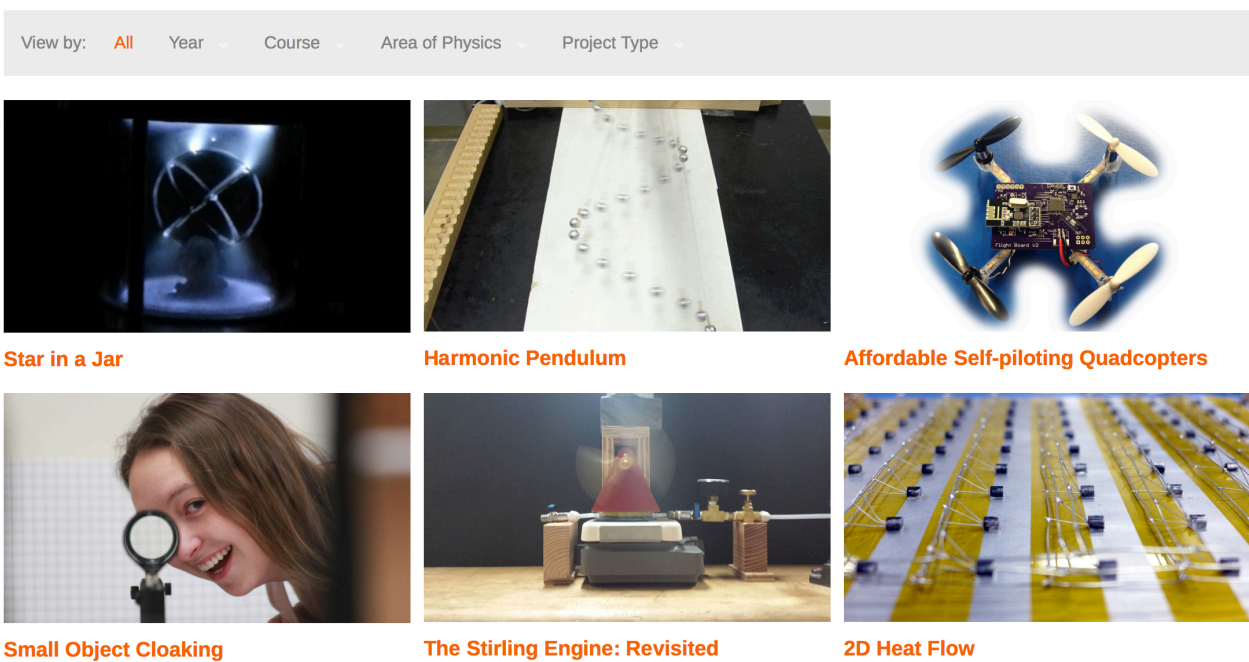

FIGURE 1. An image of $L C p h y s X$ 's Projects page. The six images shown are clickable thumbnails for student projects, each linking to an individual project page, as in Fig. 2. The "View by" labels produce drop-down menus for sorting the projects.

presented in parallel with methodologies used. Furthermore, voice-overs and images of actual scientists in the videos can counter the off-putting perception that science is unapproachable and onesided by humanizing the information, making it more accessible. $^{6}$

The project presented in this paper responds to a curricular gap in undergraduate physics by teaching students to communicate physics concepts to a popular audience using multi-media tools, specifically online video. A secondary motivation is that engaging in science communication via new media has been shown to enhance students' learning and understanding. ${ }^{7}$ A video presentation assignment was introduced to Lewis \& Clark College's (LC) Advanced Laboratory course in Spring 2015, and these student-produced videos are hosted on a new publically available website titled

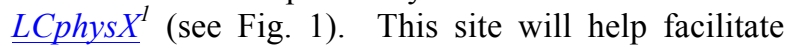
departmental outreach activities.

This paper begins with an outline of internal departmental motivations for building a digital archive of student projects, followed by a discussion of website design and functionality. We close with pedagogical applications of LCphysX, including plans for leveraging the website for public outreach and ideas for assessing its effectiveness.

\section{ARCHIVAL MOTIVATIONS FOR LCphysX}

In LC's Advanced Laboratory course, students work individually or in teams to pursue rigorous projects and experiments that align with their personal interests. Past examples include launching and recovering near-space balloons that reached $\sim 20 \mathrm{~km}$, and spectral analysis and digital recreation of bowed violin strings. Successful execution of these projects is supported by a carefully structured syllabus including a proposal and prototyping process, an allocation of departmental resources for supplies, and the support of two exceptional technical staff. For students who do not pursue a summer research experience or a Senior Thesis (an optional component of the LC physics major), their Advanced Laboratory projects serve as the main cumulative achievement of their physics education.

Modeled after a similar project documenting $\underline{\mathrm{LC}}$ Senior Art Projects ${ }^{2}, L C p h y s X$ was conceived as a permanent digital archive for student projects. Although digital records of the students' journal-style

\footnotetext{
2 https://library.lclark.edu/seniorprojects/
} 


\section{D Heat Flow}

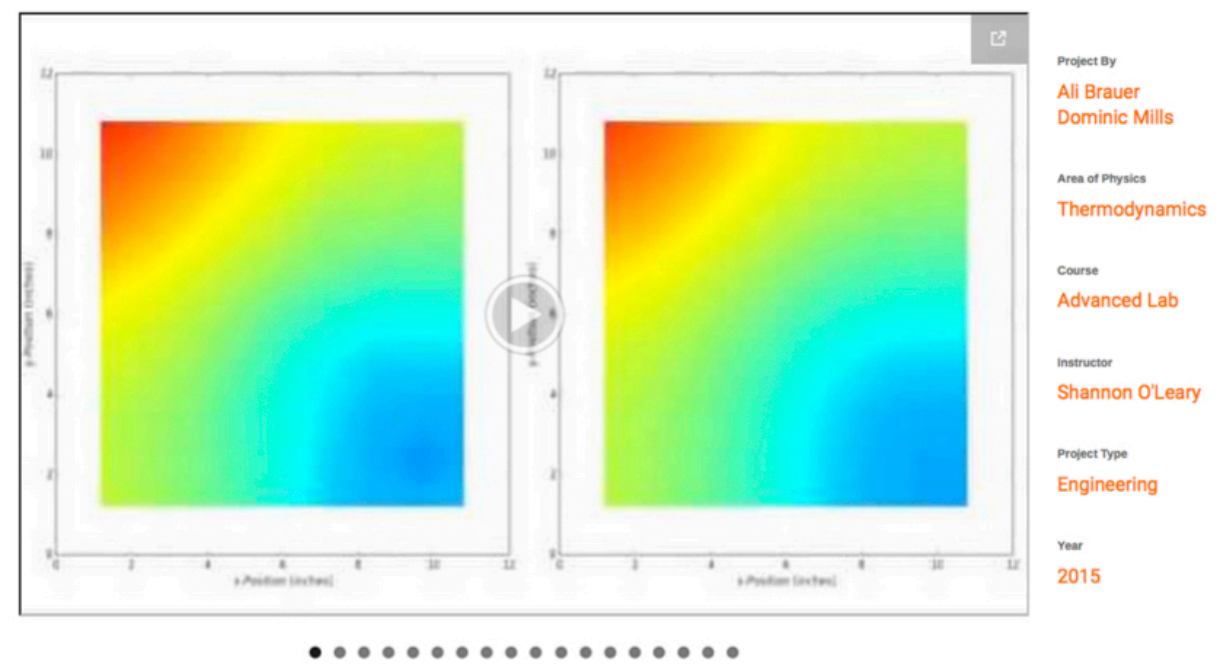

Our apparatus allows us to study two-dimensional heat flow. It consists of a square aluminum plate, 81 DS18B20 temperature sensors, and an Arduino Uno microcontroller [1]. The sensors communicate with the Arduino via a one-wire protocol, which means that all 81 sensors can be placed on a single serial

FIGURE 2. This is an image of an individual $L C p h y s X$ project on a $2 \mathrm{D}$ Heat Flow Apparatus. ${ }^{8}$ Note the (orange) tags on the right-hand side bar are clickable, bring up a Project page (as in Fig. 1) featuring thumbnails of all similarly tagged projects.

papers and colloquium presentations slides are kept on file by individual instructors, in most cases these documents do not adequately capture the projects "in action" or publically celebrate the students' achievements. Additionally, these documents are not available for browsing by other students. By compiling project descriptions on a public platform, LCphys $X$ serves as a multi-purpose departmental resource: (1) LCphys $X$ is a reference for LC students and professors engaged in new projects. (2) Because of the cumulative nature of the projects, the archive provides an on-going means for departmental assessment of the physics major as a whole. (3) As the Advanced Laboratory course is a requirement for all LC physics majors, all physics graduates leave with an active "link" for their resumés that showcases their technical achievements, as well as their ability to communicate scientific ideas. (4) With a prominent link to $L C p h y s X$ on the department website, the archive publicizes the exceptional learning opportunities available to LC Physics students (to prospective students and funders). This public information is valuable for prospective students, alumni, and potential donors. (5) Finally, this archive will be leveraged for use in public outreach activities, discussed more below.

\section{WEBSITE DESIGN AND FUNCTIONALITY}

In partnership with the LC Watzek Library Digital Initiatives team who have professional web design backgrounds, we designed LCphys $X$ using WordPress to have a clean aesthetic and intuitive functionality. The home page, accessible from all pages via the $L C p h y s X$ logo in the menu bar, welcomes the web visitor and presents images of several selected projects.

The Projects page (see Fig 1), shows a matrix of thumbnail images of student projects, each linking to an individual project page, as in Fig. 2. In order to serve as a functionally sortable archive, the individual projects are tagged according to following categories: Student Names, Area of Physics, Course, Instructor, Project Type, and Year. The "View by" bar enables the viewer to sort the projects by specific tags in each category. All text entered into LCphys $X$ is fully searchable via the menu bar magnifying glass icon.

The individual project pages, an example of which is shown in Fig. 2, feature a project video. A series of grey dots beneath the video indicate scrollable supplementary images. Accompanying the images is a short descriptive paragraph that can feature references and links to related resources. The metadata in the 
right side bar are all clickable tags that will bring up an iteration of the Project page (as in Fig. 1) featuring thumbnails of all similarly tagged projects.

\section{PEDAGOGICAL APPLICATIONS AND PUBLIC OUTREACH}

As mentioned above, a video assignment was introduced into LC's Advanced Lab course that requires students to prepare a multi-media presentation appropriate for a public audience that describes their project. Students were made aware of $L C p h y s X$, and told that best presentations would be featured on the new website. The specific format of the presentations was left open-ended, but the following elements were suggested: using photographs and videos taken throughout semester depicting progress, using presentation slides of data and analysis from their final oral presentation, taking new footage that demonstrates the most interesting aspects of their final product, and writing a voice-over script to accompany their videos, images, and graphs. In addition, the students were asked to write an abstract that succinctly but clearly describes their project in language understandable by a public audience, and to identify key still images to support their video presentation. We note that this first group of students enthusiastically embraced the assignment, and the prospect of being featured on LCphys $X$ was motivational.

As each physics major is required to take Advanced Laboratory twice, there is an expected growth between the first and second iterations in not only the sophistication of their projects, but also in their ability to communicate science to a public audience. We also expect to see the quality of the multi-media presentations improve as there are more examples available for future students to refer to and draw inspiration from.

As LCphys $X$ becomes a focal point of the $\mathrm{LC}$ Physics Department, multi-media assignments can be successfully incorporated into several other areas of the curriculum. In particular, a sophomore level Experimental Methods class features a three-week project that is ideal for such an assignment. Additionally, students who engage in Independent Study and Senior Theses will benefit from preparing a multi-media presentation appropriate for a public audience. In preparing these videos for publication on LCphys $X$, LC majors will gain an understanding and appreciation of the importance of public outreach.

Due to the public nature of the website, LCphys $X$ enables students to communicate physics concepts to community members outside the university environment. As a pilot outreach project, we plan to send out a link to $L C p h y s X$ to area high schools, with a request for feedback via a simple online survey. Schools that have high response rates could host LC physics majors for a classroom visit to answer questions and explain concepts in physics with applicable demonstrations. With the success of this initial project and as the video database of LCphys $X$ grows, we plan to contact high schools across the nation for similar feedback to the videos. Additionally, LCphys $X$ supports online comments and questions via a webform. These opportunities for dialog will enrich the experience for the undergraduate physics majors and their public audience.

\section{CONCLUSIONS}

In summary, we have developed an online platform for showcasing student-produced videos on upper-level physics projects and experiments. Through producing and publishing these videos, undergraduate physics majors will learn the importance of public outreach, and how to use new media to effectively reach a nonspecialist audience. It is also our hope that physicists and non-scientists alike will visit $L C p h y s X$, and that they find the content enriching and informative.

\section{ACKNOWLEDGMENTS}

The authors would like to acknowledge Jeremy McWilliams and Anneliese Dehner, the Digital Initiatives Team, with whom we worked in close partnership to design and implement LCphysX. We would also like to acknowledge Steve Attinasi, the LC Natural Science Shop Supervisor and Alan Younis, Scientific Electronics Specialist for their expert assistance in guiding advanced LC physics projects.

\section{REFERENCES}

1. UNESCO World Conference on Science, Declaration on Science and the Use of Scientific Knowledge, (1999).

2. L. Mercer-Mapstone and L, Kuchel, Intl. J. Sci. Ed. ahead-of-print, 37 (10), 1 (2015); S. Brownell, J. Price, and L. Steinman, J. Undgrd. Neurosci. Ed. 12, E6 (2013).

3. C. Wilcox, Bio. Bulltn. 222, 85 (2012).

4. E. Streier, N. Pfeffermann, and J. Grapp., in Strategies and Communications for Innovations, edited by $\mathrm{M}$. Hülsmann and N. Pfeffermann (Springer, Berlin, 2011) pp. 293-304.

5. J. C. Besley, and A. H. Tanner. Sci. Commun. 33, 239 (2011).

6. D. K. Smith, J. Chem. Ed. 91, 1594 (2014).

7. W. Rifkin, N. Longnecker, J. Leach, and L. Davis, in: Proceedings of The Australian Conference on Science and Mathematics 16, (2010).

8. P. McDougall and E. Ayars, Am. J. Phys. 82, 620 (2014). 